\title{
Study of the change of surface magnetic field associated with flares
}

\author{
Yixuan $\mathrm{Li}^{1}$, Ju Jing ${ }^{1}$, Yuhong Fan ${ }^{2}$ and Haimin Wang ${ }^{1}$ \\ ${ }^{1}$ Space Weather Research Lab, New Jersey Institute of Technology, Newark, USA \\ email: yl89@njit.edu, jj4@njit.edu, haimin@flare.njit.edu \\ ${ }^{2}$ High Altitude Observatory, National Center for Atmospheric Research $\dagger$, Boulder, USA \\ email: yfan@ucar.edu
}

\begin{abstract}
How magnetic field structure changes with eruptive events (e.g., flares and CMEs) has been a long-standing problem in solar physics. Here we present the analysis of eruptionassociated changes in the magnetic inclination angle, the transverse component of magnetic field and the Lorentz force. The analysis is based on an observation of the X3.4 flare on Dec.13 2006 and a numerical simulation of a solar eruption made by Yuhong Fan. Both observation and simulation show that (1) the magnetic inclination angle in the decayed peripheral penumbra increases, while that in the central area close to flaring polarity inversion line (PIL) deceases after the flare; (2) the transverse component of magnetic field increases at the lower altitude near flaring PIL after the flare. The result suggests that the field lines at flaring neutral line turn to more horizontal near the surface, that is in agreement with the prediction of Hudson, Fisher \& Welsch (2008).
\end{abstract}

Keywords. Sun: flares; sun: magnetic fields

\section{Introduction}

The 3-D magnetic topology of active regions from the photosphere to corona is of fundamental importance to our understanding of the energy storage and release process that accounts for flares and coronal mass ejections (CMEs). Recently, it has been widely reported that photosphere magnetic fields can experience some rapid, significant and permanent changes during $\mathrm{X}$-and M-class flares. In the reconnection picture proposed by Liu et al. (2005): part of the penumbral segments in the outer $\delta$ spot vanish rapidly after flares, and meanwhile, the umbral cores and/or inner penumbral regions are darkened. There are two separate magnetic systems in a $\delta$ sunspot before the flare; after the flare these two become strongly connected. The outer field lines stretched out by the eruption turn the field of the outside penumbrae to become more vertical, explaining the penumbral decay. Meanwhile, the lower field lines near the neutral line (NL) become more horizontal, and thus explain the darkening of the central umbral regions.

Although the flare-associated magnetic changes have been reported, the flare models which could reveal the physical mechanism for these changes have not been achieved. Part of the reason for this is that we can only make observations of the solar magnetic fields directly and precisely at the photosphere level. A good understanding of the role that the magnetic fields play in powering the flares is essentially based on study the 3-D structure of magnetic fields and their evolution associated with flares. We will compare

$\dagger$ The National Center for Atmospheric Research is Sponsored by the National Science Foundation 
our observational findings with numerical simulations of eruptive flares to examine how the observational signatures compare with the predictions of the specific models.

\section{Data sets and model description}

The data base for this paper consists of the X3.4 flare occurred in NOAA active region 10930 on 2006 December 13 and a numerical simulation of an eruption in the solar corona in a spherical domain (Fan 2010).

For this X3.4 event, we used the Hinode vector magnetograms taken before and after the 4B/X3.4 flare to study the changes of magnetic field. The vector magnetograms were obtained in two time bins, 20:30-21:33 UT on 2006 December 12 (before the flare) and 4:30-5:36 UT on 2006 December 13 (after the flare). The pixel resolution of the magnetograms is 0.16 " pixel $^{-1}$. Combined with the corresponding G-band (430nm) data, we identified the decayed and the enhanced regions of the spot. Since the active region is not located at the center of solar disk, the projection effect was corrected with coordinate transformation. The $180^{\circ}$ ambiguity in vector magnetograms was resolved with the "minimum energy" method (Metcalf 1994).

For the numerical simulation shown in this letter, the detailed magnetic field structure was obtained by solving the isothermal MHD equations in a spherical domain representing the solar corona, given by $r \in\left[R_{\odot}, 5.496 R_{\odot}\right], \theta \in[5 \pi / 12,7 \pi / 12], \phi \in[-\pi / 9.6, \pi / 9.6](\mathrm{Fan}$ 2010). The simulated domain has been resolved in $432 \times 192 \times 240$ grids, which is uniform in $\theta$ and $\phi$ direction, and non-uniform along the solar radius, and the step size in the range from $r=R_{s}$ to $r=1.788 R_{s}$ is $d r=0.0027271 R_{s}$. The initial state of this simulation is assumed to contain a pre-existing potential arcade field in a hydrostatic isothermal atmosphere domain. The emergence of a twisted and arched flux tube is imposed at the lower boundary with an upward advection velocity $\mathbf{v}_{0}$ (small compared to the Alfvén speed) in the area where the emerging tube intersects the lower boundary. After the emergence is stopped, a quasi-equilibrium of coronal flux rope with an underlying sigmoid shaped current sheet is established immediately. Subsequently, the flux rope continued to build up due to reconnections in the current sheet and the ejective eruption is triggered when flux rope exceeds a critical height. In this study, we concentrate on the evolution of the magnetic field immediately above the line-tying lower boundary, at the grid level 2 .

\section{Calculation of lorentz force change}

Given a change $\delta \mathbf{B}$ in the photospheric magnetic field, Hudson et al. (2008) predicted the back reaction in the photosphere by estimating the change in Lorentz force per unit area as $\delta f_{z}=\left(B_{z} \delta B_{z}-B_{x} \delta B_{x}-B_{y} \delta B_{y}\right) / 4 \pi$. We calculate the change of Lorentz force by integrating the $\delta f_{z}$ over an area at flaring PIL.

\section{Results}

For this X3.4 event, we compare the sunspot structures before and after the flare in Figure 1. The brightened/darkened areas in the difference image represent the penumbral regions with decayed/enhanced G-band intensity. The right two panels show the time evolution of the mean G-band intensity in the brightened area (top) and the darkened area (bottom) during a $3.5 \mathrm{hr}$ time period around the flare.

After aligning the G-band images with the corresponding SP vector magnetograms, we are able to study the changes of the photospheric magnetic parameters in the decayed and 

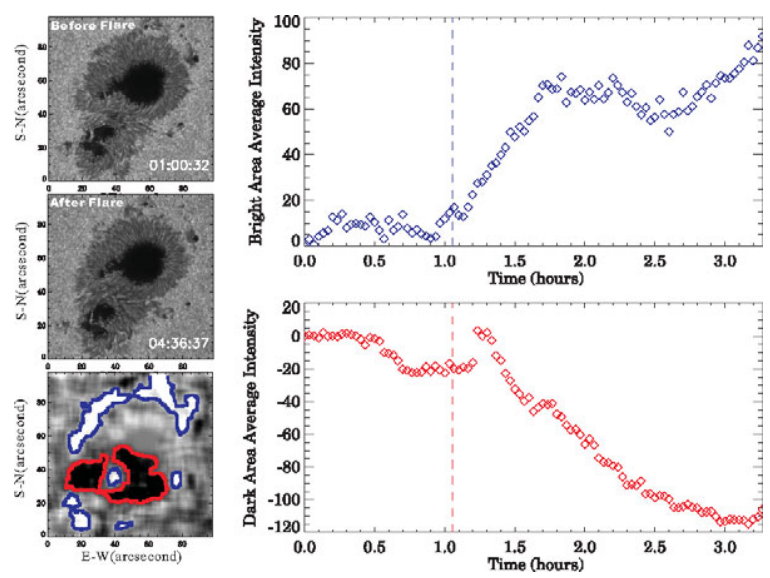

Figure 1. The left column shows Hinode G-band images taken before (top) and after the flare (middle), and their difference image (the post-flare image subtracted by the pre-flare image; bottom). The field-of-view (FOV) is $100 " \times 100$ ". In the difference image, the brightened area and the darkened area are outlined with blue and red contours, respectively. In the right column, Top Panel: Evolution of the mean G-band intensity of the decayed region, starting from 01:10 December 13, 2006;Bottom Panel: Evolution of the mean G-band intensity of the enhanced region. The dashed lines indicate the flaring peak time of hard X-rays.

enhanced penumbral areas. Figure 2 compares the pre-flare and the post-flare distributions of magnetic inclination angle, transverse field strength in the decayed (left column) and the enhanced (right column) areas. The mean values of parameters before and after the flare are indicated by the vertical green and orange lines, respectively. Evidently we can see that the inclination angle increases by $\sim 3.3^{\circ}$ after the flare in the decayed region, and decreases by $5^{\circ}$ in the enhanced region. The transverse magnetic field strength within the centered enhanced region increased by $20 \%$ after the flare, meanwhile which falls the amount of $16 \%$ in the peripheral decayed region. In this case, it demonstrates that the magnetic fields change from a more inclined to a more vertical configuration in the peripheral penumbral region.

As a comparison, we have analyzed a sequence of the MHD simulations. In Figure 3, the left two columns compare the magnetic transverse field and Light-of-sight(LOS) field structure taken before and after the eruption in the second layer of grid along $r$, which is about $4.745 \mathrm{Mm}$ above the solar surface. The decayed and enhanced areas are represented by the red and blue boxes. The blue boxes contour the enhanced areas after eruption, which correspond to the darkening inner penumbra, whereas the red boxes correspond to the outer decaying penumbra. The right panels (from top to bottom) show respectively the time profile of magnetic inclination angle, the transverse field strength and the change of Lorentz force comparing to $t=150$ (the unit for time is $\tau=356.8 \mathrm{~s}$ ). The dashed lines indicate the flaring time. We can see that the inclination angle increases and the $B_{t}$ decreases after the flare in the outer decayed region, while the inclination angle decreases and the $B_{t}$ increases at flaring neutral line. The sudden enhancement of the downward Lorentz force after the flare, which may indicate the back reaction on the photosphere and solar interior due to releasing of flare energy, is consistent with the prediction by Hudson, Fisher \& Welsch.

We note that Moore et al. (2001) proposed the Tether-cutting model to explain the onset of flares and subsequent eruptions. In this reconnection model, a short and flat loop forms near the photosphere after the eruption, which is consistent with our observation 

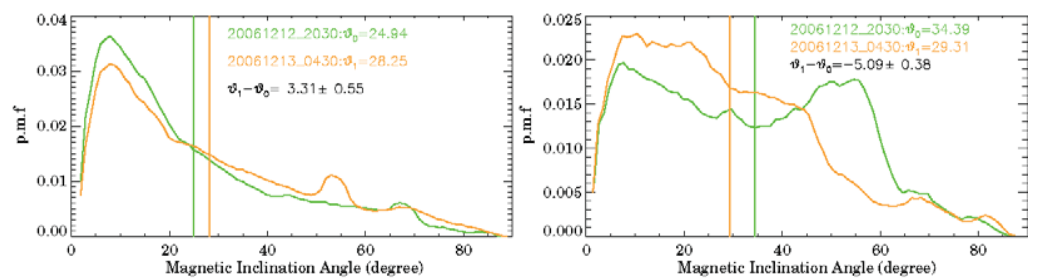

(a) Magnetic Inclination Angle
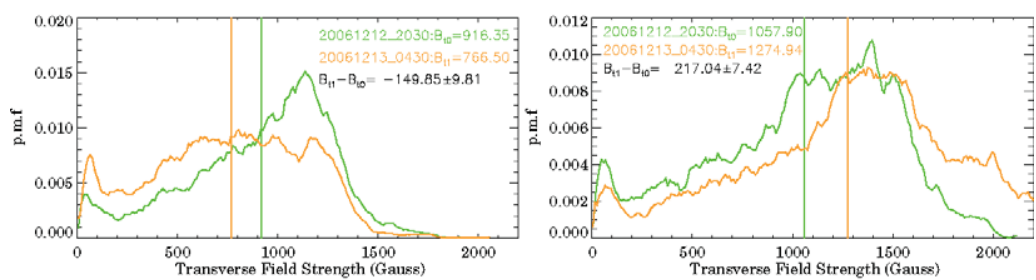

(b) Transverse Field Strength

Figure 2. The pre-flare (green lines) and the post-flare (orange lines) distributions of magnetic inclination angle, transverse field strength in the decayed (left column) and the enhanced (right column) areas. The vertical green lines indicate the mean value of parameters before the flare, while the vertical orange lines indicate that after the flare. The $95 \%$ confidence intervals of each parameter were shown in corresponding panel.
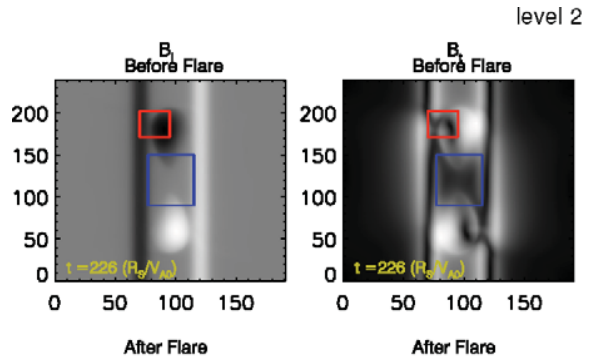

level 2
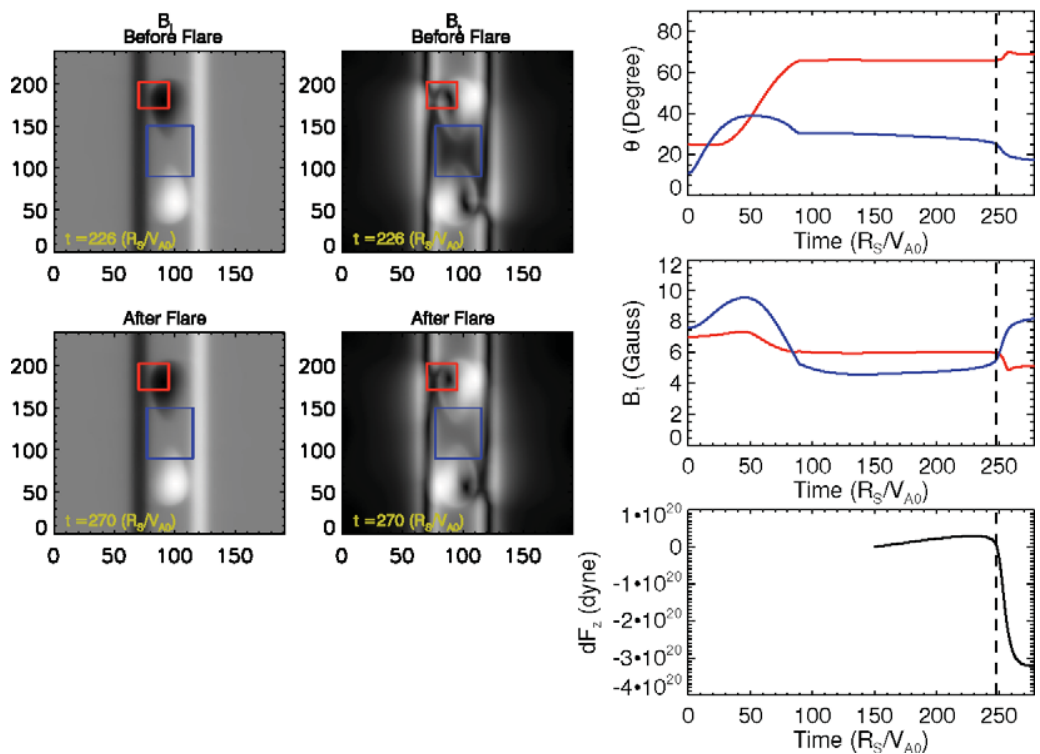

Figure 3. Left two columns: The magnetic transverse fields and LOS fields before and after the eruption with the red and blue boxes indicated the decayed and enhanced regions, respectively. Right column: The time profile, from top to bottom, of magnetic inclination angle, the transverse field strength and the change of Lorentz force comparing that at $t=150 \tau$. 
of the enhanced transverse field near flaring NLs. This is an example of predicting permanent field changes associated with flares, also consistent with our observational and simulated results.

This work was supported by NSF grant AGS-0936665 and NASA grants NNX08BA22G and NNX08-AQ90G to New Jersey Institute of Technology.

\section{References}

Hudson, H. S., Fisher, G. H., \& Welsch, B. T. 2008, Subsurface and Atmospheric Influences on Solar Activity, 383, 221

Liu, C., Deng, N., Liu, Y., Falconer, D., Goode, P. R., Denker, C., \& Wang, H. 2005, Astrophys. J., 622,722

Fan, Y. 2010, Astrophys. J., 719, 728

Metcalf, T. R. 1994, Solar Phys., 155, 235

Moore, R. L., Sterling, A. C., Hudson, H. S., \& Lemen, J. R. 2001, Astrophys. J., 552, 833 